\title{
Long-term survival and prognostic factors of five-year survivors with complete resection of non-small cell lung carcinoma
}

\author{
Morihito Okada, MD, PhD \\ Wataru Nishio, MD, PhD \\ Toshihiko Sakamoto, MD, PhD \\ Hiroaki Harada, MD \\ Kazuya Uchino, MD \\ Noriaki Tsubota, MD, PhD
}

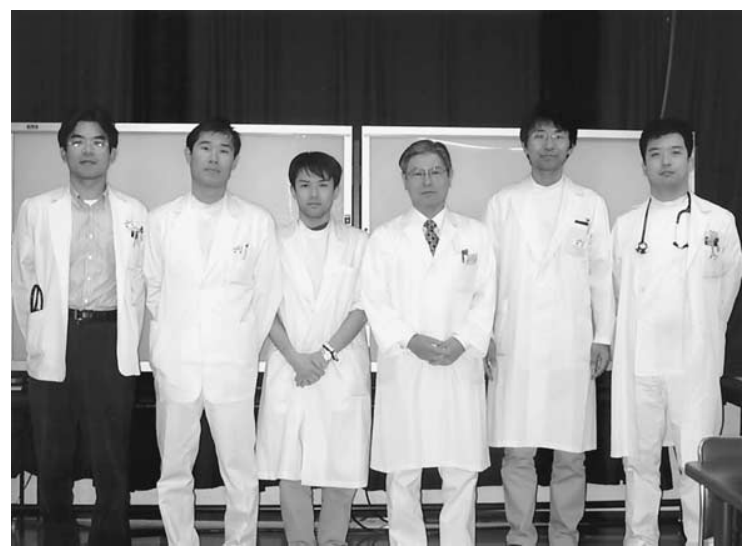

Sakamoto, Nishio, Uchino, Tsubota, Okada, and Harada (left to right)
Objective: We analyzed the long-term follow-up data on cancerrelated death in 5-year survivors of complete resection of their non-small cell lung cancer and examined the prognostic factors having an impact on subsequent survival.

Methods: Of 848 consecutive patients with proven primary nonsmall cell carcinoma who underwent complete removal of the primary tumor together with hilar and mediastinal lymph nodes, 421 patients $(49.6 \%)$ survived 5 years or longer after the initial surgical treatment. Of all the data analyzed, only death related to cancer was treated as death.

Results: The median follow-up of 5-year survivors was 84 months from the original treatment (range, 60 to 200 months). Their overall survival rate at 10 years was $91.0 \%$. Multivariable Cox analysis demonstrated that although advanced surgical-pathological stage $(P$ $=.0001)$, nodal involvement $(P=.0245)$, male gender $(P=.0313)$, and nonsquamous type of the tumor $(P=.0034)$ were significant, independent, unfavorable prognostic determinants in all patients, none of the variables investigated significantly influenced the long-term survival of 5-year survivors. The rate of recurrence beyond 5 years was much lower compared with that within 5 years. In contrast, the rate of occurrence of new malignancies was unchanged throughout the long-term postoperative period.

Conclusions: Among 5-year survivors of complete resection of non-small cell lung cancer, neither stage, nodal status, sex, nor histologic condition further affected subsequent survival, suggesting that the 5-year interval might be sufficient to declare that a patient with lung cancer has been cured.

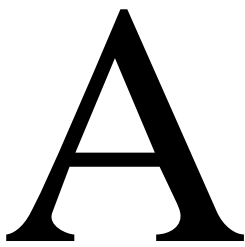

lthough pulmonary resection remains the most effective treatment for patients with non-small cell lung cancer who are presumed to have no distant metastases and no lymph node involvement beyond the resected specimen, a considerable number of these patients have recurrent lung cancer within 5 years. Generally, the success or failure of therapeutic intervention including surgery in patients with cancer has been assessed in time of survival, often with emphasis on the first 5 years subsequent to the intervention. Despite our expectation of long-term survival after complete resection of non-small cell lung cancer, recurrence can 
develop in one fourth of the patients, even of those with stage IA disease. ${ }^{1-3}$ Therapeutic success can be overestimated by survival rates restricted to the initial 5 years if the risk of recurrence 5 years or later is substantial. ${ }^{4,5}$ There are many articles regarding the natural history of patients with resected non-small cell lung cancer during the early postoperative period but few about the long-term survival of such patients, in particular beyond 5 years. The purposes of this study were to analyze the long-term follow-up data on cancer-related death in patients after complete resection of non-small cell lung cancer and to determine the significant prognostic factors in patients alive 5 years from surgical treatment of their initial cancer.

\section{Patients and Methods}

Between July 1984 and July 1997, 1060 consecutive patients were operated on for primary lung cancer by the same surgical team. Of the 1060 patients, 1031 patients were histologically diagnosed as having non-small cell carcinoma, and 183 patients could not undergo complete resection nor nodal dissection. During that period, 848 patients with proven non-small cell carcinoma underwent complete removal of the primary tumor together with ipsilateral hilar and mediastinal lymph nodes. Of them, 421 patients (49.6\%) survived 5 years or longer. Patients who had evidence of residual tumor at the surgical margin, malignant effusion, or N3 disease verified by intraoperative findings or postoperative pathologic examination were defined as having been subjected to an incomplete surgery and were excluded from this study. The following criteria, based on and modified from those of Martini and Melamed, ${ }^{6}$ have been used for the designation of multiple primary lung cancers ${ }^{7}$ : the tumors were anatomically separate, and the tumors were histologically different. If the tumors were histologically the same, they must have no systemic metastases or mediastinal spread. In case of metachronous tumors with the same histology, tumor-free interval should be at least 2 years. Tumors with minute satellite nodules that were found incidentally within the same lobe of the resected specimen were not excluded from this study, because we were not certain whether these lesions should be considered local tumor spread or not. Tumors with satellite lesions in another lobe were excluded. Patients whose tumors were subsequently classified as small cell carcinoma or low-grade malignant tumor were also excluded. Surgical-pathologic staging was carried out according to the New International Staging System for Lung Cancer. ${ }^{8}$

Routine systematic dissection of all the hilar and mediastinal nodes was performed in every case, even if the preoperative evaluation was N0 or N1. ${ }^{9}$ Every node dissected was examined by pathologists to be diagnosed as microscopically positive or negative during and after the operation. No adjuvant therapies were provided before or after surgery.

After surgery, the patients were examined at 3-month intervals for 5 years and thereafter at 1-year intervals in general. The evaluations included physical examination, chest roentgenography, and tumor markers. Moreover, chest, abdominal, and brain computed tomographic scans and a bone scintiscan were carried out each year. Whenever any symptoms or signs of recurrence
TABLE 1. Histologic type

\begin{tabular}{lc}
\hline Cell type & Number (\%) \\
\hline Adenocarcinoma & $245(58.2)$ \\
Squamous cell carcinoma & $160(38.0)$ \\
Adenosquamous carcinoma & $8(1.9)$ \\
Large cell carcinoma & $8(1.9)$ \\
Total & $421(100)$ \\
\hline
\end{tabular}

TABLE 2. Extent of pulmonary resection

\begin{tabular}{lc}
\hline Procedure & Number (\%) \\
\hline Lobectomy & \\
Lobe only & $295(70.1)$ \\
$\quad+$ Sleeve & $36(8.6)$ \\
Segmentectomy & \\
Segment only & $62(14.7)$ \\
$\quad+$ Sleeve & $4(1.0)$ \\
Pneumonectomy & \\
Unilateral lung & $13(3.1)$ \\
$\quad+$ Sleeve & $1(0.2)$ \\
Wedge resection & $10(2.4)$ \\
Total & $421(100)$ \\
\hline
\end{tabular}

TABLE 3. Surgical-pathological staging

\begin{tabular}{lccr}
\hline & & \multicolumn{2}{c}{ Number (\%) } \\
\cline { 3 - 4 } Stage & & All patients & $\begin{array}{c}\text { 5-year } \\
\text { survivors }\end{array}$ \\
\hline IA & T1 N0 & $285(33.6)$ & $181(43.0)$ \\
IB & T2 N0 & $200(23.6)$ & $109(25.9)$ \\
IIA & T1 N1 & $36(4.2)$ & $25(5.9)$ \\
IIB & T2 N1 & $64(7.5)$ & $25(5.9)$ \\
& T3 N0 & $45(5.3)$ & $22(5.2)$ \\
IIIA & T3 N1 & $26(3.1)$ & $7(1.7)$ \\
& T1 N2 & $33(3.9)$ & $16(3.8)$ \\
& T2 N2 & $73(8.6)$ & $11(2.6)$ \\
IIIB & T3 N2 & $36(4.2)$ & $5(1.2)$ \\
& T4 N0 & $16(1.9)$ & $11(2.6)$ \\
& T4 N1 & $15(1.8)$ & $7(1.7)$ \\
Total & T4 N2 & $19(2.2)$ & $2(0.5)$ \\
& & $848(100)$ & $421(100)$
\end{tabular}

appeared in these examinations, further evaluations to detect the disease were performed.

Survival was calculated by the Kaplan-Meier method, and differences in survival were determined by the log-rank analysis. A multivariable analysis of several independent prognostic factors was carried out using Cox's proportional hazards regression model. Zero time was the date of pulmonary resection, and the terminal event was death attributable to cancer. To avoid controversy, we defined death as a cancer-related death when recurrence was evident before death or at autopsy. All other deaths by noncancer or unknown causes were treated as withdrawals, which means that death that was not caused by cancer was considered censored. Significance was defined as $P<.05$. 
TABLE 4. Multivariable analysis of prognostic factors among patients after complete resection of non-small cell lung cancer $(\mathbf{n}=848)$

\begin{tabular}{lccccc}
\hline Factors & Unfavorable & Favorable & Risk ratio & $\mathbf{9 5 \%}$ Cl & $\boldsymbol{P}$ value \\
\hline $\mathrm{pN}$ & $\mathrm{N} 1+\mathrm{N} 2$ & $\mathrm{~N} 0$ & 1.570 & $1.060-2.326$ & .0245 \\
$\mathrm{p}$-stage & $\mathrm{II}+\mathrm{III}$ & $\mathrm{I}$ & 2.627 & $1.745-3.954$ & .0001 \\
Age & $65 \leq$ & $65>$ & 1.222 & $0.966-1.545$ & .0952 \\
Sex & $\mathrm{Male}$ & Female & 1.379 & $1.029-1.848$ & .0313 \\
Histologic type & $\mathrm{Non}-\mathrm{SO}$ & $\mathrm{SO}$ & 1.455 & $1.132-1.869$ & .0034 \\
Extent of procedure & $\mathrm{Pn}+\mathrm{Lo}$ & $\mathrm{Se}+\mathrm{We}$ & 1.225 & $0.836-1.795$ & .2977
\end{tabular}

$\mathrm{Cl}$, Confidence interval; SQ, squamous cell carcinoma; $P n$, pneumonectomy; Lo, lobectomy; Se, segmentectomy; We, wedge resection.

TABLE 5. Multivariable analysis of prognostic factors among patients alive 5 years after complete resection of non-small cell lung cancer $(n=421)$

\begin{tabular}{|c|c|c|c|c|c|}
\hline Factors & Unfavorable & Favorable & Risk ratio & $95 \% \mathrm{Cl}$ & $P$ value \\
\hline $\mathrm{pN}$ & $\mathrm{N} 1+\mathrm{N} 2$ & No & 1.421 & $0.344-5.865$ & .6275 \\
\hline p-stage & $\mathrm{II}+\mathrm{III}$ & I & 2.949 & $0.806-10.784$ & .1021 \\
\hline Age & $65 \leq$ & $65>$ & 1.441 & $0.575-3.615$ & .4357 \\
\hline Sex & Female & Male & 1.469 & $0.569-3.791$ & .4269 \\
\hline Histologic type & Non-SQ & SQ & 2.910 & $0.908-9.326$ & .0722 \\
\hline Extent of procedure & $\mathrm{Pn}+\mathrm{Lo}$ & $\mathrm{Se}+\mathrm{We}$ & 1.141 & $0.253-5.151$ & .8637 \\
\hline
\end{tabular}

$C l$, Confidence interval; $S Q$, squamous cell carcinoma; $P n$, pneumonectomy; Lo, lobectomy; Se, segmentectomy; We, wedge resection.

\section{Results}

There were 291 men and 130 women with a mean age of 62.8 years (range, 30 to 82 years). Overall follow-up ranged from 60 to 200 months, with a median of 84 months. The histologic classification was adenocarcinoma in $58.2 \%$ of the patients, squamous cell carcinoma in $38.0 \%$, adenosquamous carcinoma in $1.9 \%$, and large cell carcinoma in $1.9 \%$ (Table 1). Lobectomy was by far the most common procedure and was performed in 331 patients $(78.7 \%)$, including 36 combined sleeve resections of bronchi. Sixty-six segmentectomies (4 bronchoplasties and 14 pneumonectomies including 1 sleeve technique for the tracheobronchus) were carried out (Table 2). As we followed the policy that lungsaving procedures must always be kept in mind, ${ }^{10,11}$ the ratio of bronchoplasties performed was relatively high $(9.8 \%)$, and that of pneumonectomies was very low. On the basis of the international staging system, $68.9 \%$ of 5 -year survivors had stage I disease, $17.0 \%$ had stage II disease, and $14.1 \%$ had stage III disease, although among all patients subjected to complete resection, $57.2 \%$ had stage I disease, $17.0 \%$ had stage II disease, and $25.8 \%$ had stage III disease (Table 3).

Multivariable Cox analysis of all patients $(\mathrm{n}=848)$ subjected to complete resection for non-small cell lung cancer demonstrated that surgical-pathological stage, nodal involvement, sex, and histologic type of the tumor were significant prognostic determinants (Table 4). However, multivariable analysis of patients who were alive 5 years after surgery showed that none of the variables examined significantly influenced additional survival (Table 5). The overall survival of the patients who had survived 5 years postoperatively was $91.0 \%$ at 10 years (Figure 1). The possibilities for patients who were alive 5 years after complete resection of lung cancer to survive beyond 5 years were excellent irrespective of the surgical-pathological stage (Figure 2) or the nodal status (Figure 3), although patients with a more advanced stage or nodal status tended to have worse prognosis.

It is interesting to note that the long-term prognosis of 5 -year survivors with non-squamous cell carcinoma was worse (not significantly, $P=.0574$ ) than that of 5-year survivors with squamous cell carcinoma (Figure 4). The histopathologic difference in survival might suggest that in some cases adenocarcinoma could progress slowly and result in recurrence beyond 5 years after complete removal of the tumor. Moreover, although the rate of late recurrence was much lower in patients alive for 5 years postoperatively, the occurrence rate for new malignancies remained unchanged throughout the long-term follow-up period (Figure 5).

\section{Discussion}

One of the most important findings in this study was that advanced stage, high involvement of lymph nodes, male gender, and non-squamous cancer were independent, unfavorable prognostic factors in all patients, but in 5-year survivors, neither stage, nodal status, gender, nor histologic condition further affected the subsequent survival, indicating that once the patients with complete resection of nonsmall cell lung cancer survive beyond 5 years, there is no 


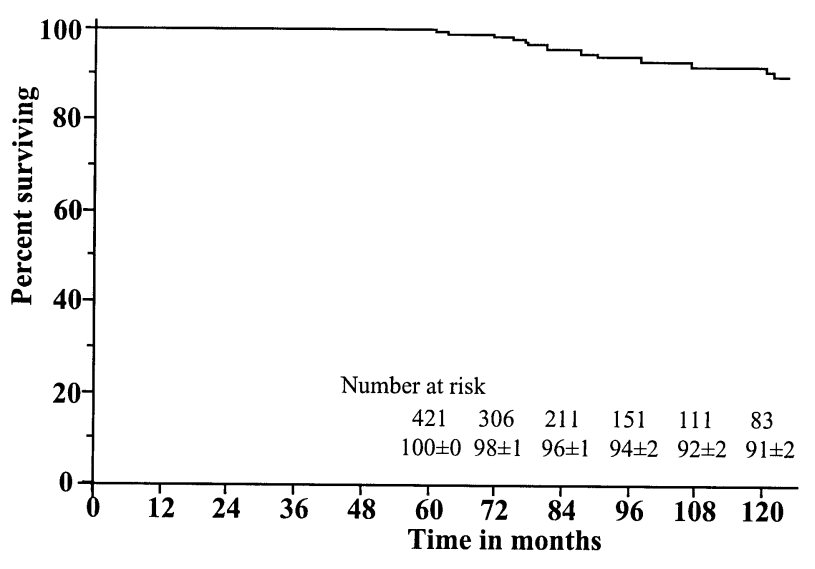

Figure 1. Cumulative survival curves of patients alive 5 years after complete resection of non-small cell lung cancer.

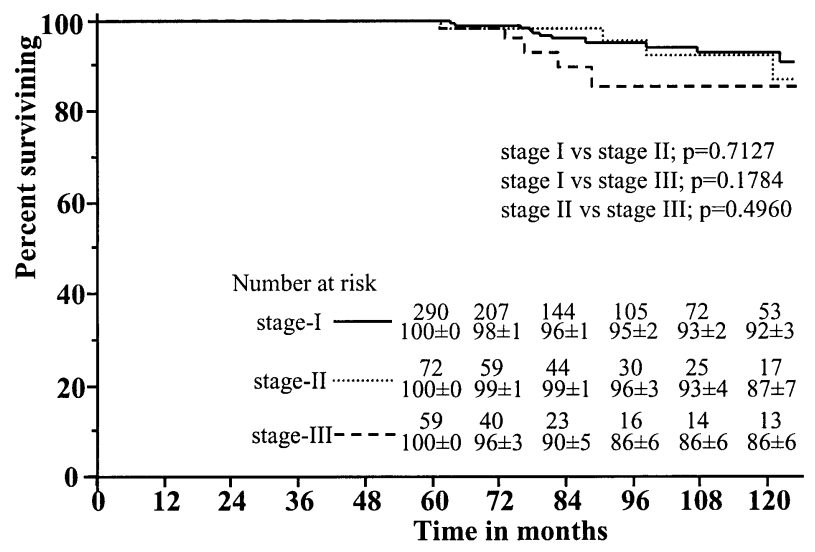

Figure 2. Cumulative survival curves of patients alive 5 years after complete resection of non-small cell lung cancer according to surgical-pathological stage.

difference in survival concerning their clinicopathologic factors (that is, the disease behaves the same after 5 years). It must be emphasized that there were so few cancer-related deaths beyond 5 years that it is possible that the test used for statistic analysis was underpowered. It is of great interest to note that although female patients had a significantly better prognosis than male patients after surgery, 5-year female survivors had a relatively worse prognosis beyond 5 years, suggesting that in some female patients adenocarcinoma might tend to progress slowly and to recur late after surgery.

There are some cases in which we can completely resect a tumor with involvement of the mediastinal nodes (N2) or direct extension into the adjacent structures (T3 to 4). In this study, 34 patients with $\mathrm{N} 2$ disease (T1 to 4 ) and 20 patients with T4 tumor were alive at 5 years from their initial surgical treatment. In the present review, we found an interesting case in which recurrence developed 6 years after resection of a T2 N2 M0 squamous cell carcinoma and was

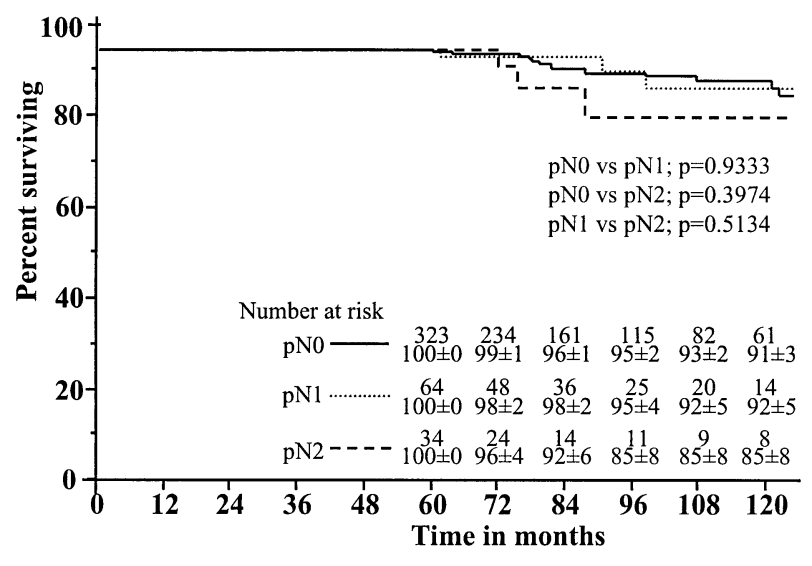

Figure 3. Cumulative survival curves of patients alive 5 years after complete resection of non-small cell lung cancer according to involvement of lymph nodes.

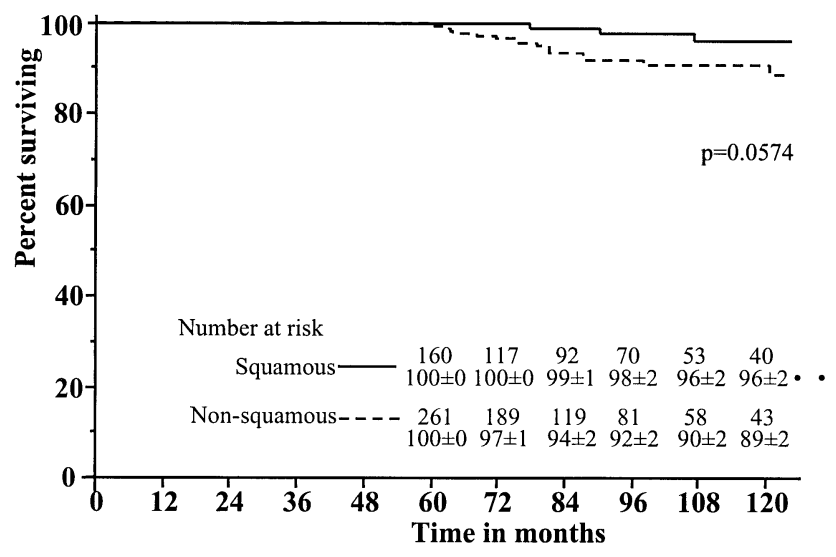

Figure 4. Cumulative survival curves of patients alive 5 years after complete resection of non-small cell lung cancer according to histologic type of the tumor.

completely removed by surgery. The patient is still alive, although 9 years have passed since the second surgery.

Despite presumed effective surgical treatment that is considered best for locally located non-small cell lung cancer, we are faced with failure to control the cancer because of distant metastases, more so in the case of patients with more advanced stage disease. Nevertheless, the patients who remain alive by 5 years after surgery appear to have a favorable outlook because $91 \%$ of these patients do not die of lung cancer thereafter. There is considerable controversy regarding when we can consider a patient with non-small cell lung cancer to have been cured. The Lung Cancer Study Group emphasized the need for continued surveillance for the survivors at 5 years after surgery because second primary cancers might recur after 5 years. ${ }^{5,12}$ In this study, the frequency of local pulmonary recurrence after 5 years was 3\%, and that of distant metastases was $6 \%$. 


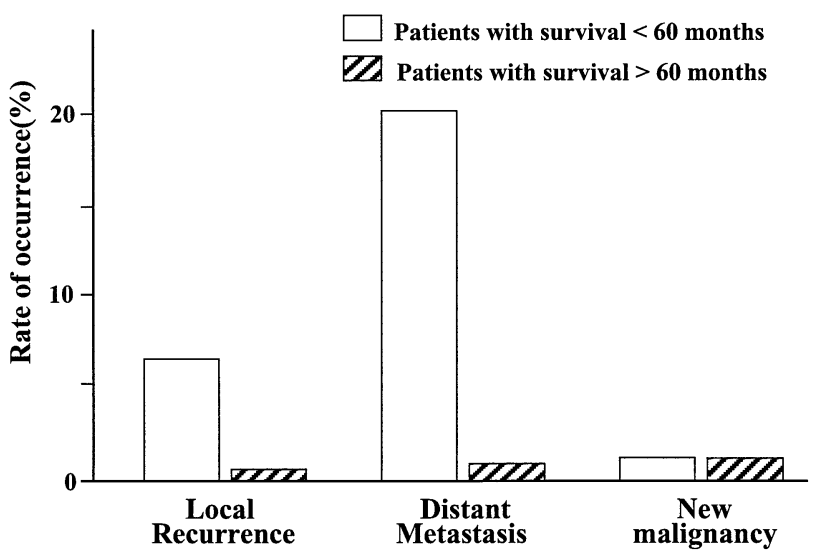

Figure 5. Comparison of rates of recurrences and new malignancies between patients who died of cancer within 5 years and those alive 5 years after complete resection of non-small cell lung cancer. Rates of occurrence were calculated as occurrences/eligible patient-year.

Although second primary cancers for which incidence remained unchanged throughout the long follow-up period continued to pose a threat, the recurrence rate decreased with time. Beyond 5 years, the low incidence of recurrence as well as survival data unrelated to the stage determined at the initial surgery are important, suggesting the 5-year interval is sufficient to declare that a patient with lung cancer has been cured.

\section{References}

1. Battafarano RJ, Piccirillo JF, Meyers BF, et al. Impact of comorbidity on survival after surgical resection in patients with stage I non-small cell lung cancer. J Thorac Cardiovasc Surg. 2002;123:280-7.

2. Martini N, Bains MS, Burt ME, et al. Incidence of local recurrence and second primary tumors in resected stage I lung cancer. J Thorac Cardiovasc Surg. 1995;109:120-9.

3. Martini N, Rusch VW, Bains MS, et al. Factors influencing ten-year survival in resected stages I to IIIa non-small cell lung cancer. $J$ Thorac Cardiovasc Surg. 1999;117:32-6.

4. Okada M, Yoshikawa K, Hatta T, Tsubota N. Is segmentectomy with lymph node assessment an alternative to lobectomy for non-small cell lung cancer of $2 \mathrm{~cm}$ or smaller? Ann Thorac Surg. 2001;71:956-60.

5. Thomas PA Jr, Rubinstein L. Malignant disease appearing late after operation for T1 N0 non-small-cell lung cancer. The Lung Cancer Study Group. J Thorac Cardiovasc Surg. 1993;106:1053-8.

6. Martini N, Melamed MR. Multiple primary lung cancers. J Thorac Cardiovasc Surg. 1975;70:606-12.

7. Okada M, Tsubota N, Yoshimura M, Miyamoto Y. Operative approach for multiple primary lung carcinomas. J Thorac Cardiovasc Surg. 1998;115:836-40.

8. Mountain CF. Revisions in the International System for Staging Lung Cancer. Chest. 1997;111:1710-7.

9. Okada M, Tsubota N, Yoshimura M, Miyamoto Y. Proposal for reasonable mediastinal lymphadenectomy in bronchogenic carcinomas: role of subcarinal nodes in selective dissection. J Thorac Cardiovasc Surg. 1998;116:949-53.

10. Okada M, Tsubota N, Yoshimura M, et al. Extended sleeve lobectomy for lung cancer: the avoidance of pneumonectomy. J Thorac Cardiovasc Surg. 1999;118:710-3.

11. Okada M, Yamagishi H, Satake S, et al. Survival related to lymph node involvement in lung cancer after sleeve lobectomy compared with pneumonectomy. J Thorac Cardiovasc Surg. 2000;119:814-9.

12. Thomas P, Rubinstein L. Cancer recurrence after resection: T1 N0 non-small cell lung cancer. Lung Cancer Study Group. Ann Thorac Surg. 1990;49:242-6. 